\title{
Autophagy: a new target for nonalcoholic fatty liver disease therapy
}

This article was published in the following Dove Press journal:

Hepatic Medicine: Evidence and Research

24 March 2016

Number of times this article has been viewed

\section{Yuqing Mao' \\ Fujun $\mathrm{Yu}^{\prime}$ \\ Jianbo Wang ${ }^{2}$ \\ Chuanyong Guo ${ }^{3}$ \\ Xiaoming Fan'}

'Department of Gastroenterology and Hepatology, Jinshan Hospital of Fudan University, Shanghai, ${ }^{2}$ Department of Gastroenterology and Hepatology, The Central Hospital of Lishui City, Wenzhou Medical University, Zhejiang, ${ }^{3}$ Department of Gastroenterology and Hepatology, Shanghai Tenth People's Hospital, Tongji University, Shanghai, People's Republic of China
Correspondence: Chuanyong Guo Department of Gastroenterology and Hepatology, Shanghai Tenth People's Hospital, Tongji University, 302 Yanchang Road, Jingan District, Shanghai 200072,

People's Republic of China

Tel +86 2I 66302535

Fax +86 21 66303983

Email guochuanyong@hotmail.com

\section{Xiaoming Fan}

Department of Gastroenterology and Hepatology, Jinshan Hospital of Fudan University, I 508 Longhang Road, Jinshan District, Shanghai 20I508,

People's Republic of China Tel +86 2I 34189990

Fax +86 2I 5794 3।4I

Email xiaomingfan57@hotmail.com

\begin{abstract}
Nonalcoholic fatty liver disease (NAFLD) has gained importance in recent decades due to drastic changes in diet, especially in Western countries. NAFLD occurs as a spectrum from simple hepatic steatosis, steatohepatitis to cirrhosis, and even hepatocellular carcinoma. Although the molecular mechanisms underlying the development of NAFLD have been intensively investigated, many issues remain to be resolved. Autophagy is a cell survival mechanism for disposing of excess or defective organelles, and has become a hot spot for research. Recent studies have revealed that autophagy is linked to the development of NAFLD and regulation of autophagy has therapeutic potential. Autophagy reduces intracellular lipid droplets by enclosing them and fusing with lysosomes for degradation. Furthermore, autophagy is involved in attenuating inflammation and liver injury. However, autophagy is regarded as a double-edged sword, as it may also affect adipogenesis and adipocyte differentiation. Moreover, it is unclear as to whether autophagy protects the body from injury or causes diseases and even death, and the association between autophagy and NAFLD remains controversial. This review is intended to discuss, comment, and outline the progress made in this field and establish the possible molecular mechanism involved.
\end{abstract}

Keywords: nonalcoholic fatty liver disease, autophagy, steatosis, steatohepatitis, fibrosis, carcinogenesis

\section{Introduction}

Nonalcoholic fatty liver disease (NAFLD) is the most common hepatic disease in Western countries, and is defined as evidence of hepatic steatosis, either by imaging or histology, with no cause for secondary hepatic fat accumulation such as significant alcohol consumption, use of steatogenic medication, or hereditary disorders. ${ }^{1}$ The prevalence of NAFLD is $20 \%-30 \%$ in Western countries, ${ }^{2}$ which causes considerable concern with its progression from steatosis to steatohepatitis, cirrhosis, and even hepatocellular carcinoma (HCC; Figure 1). NAFLD is associated with hepatic insulin resistance and type 2 diabetes. ${ }^{3}$ Recent studies have revealed that patients with NAFLD are at a higher risk of developing coronary atherosclerosis, which might be an independent risk factor. ${ }^{4}$ The mechanisms involved in the onset of NAFLD are complicated and are not entirely clear at present. Recent advances in the understanding of the molecular processes contributing to autophagy have provided insight into the relationship between autophagy and NAFLD. Autophagy might stimulate lipid metabolism, ${ }^{5}$ and thus has therapeutic potential in NAFLD. ${ }^{6}$ 


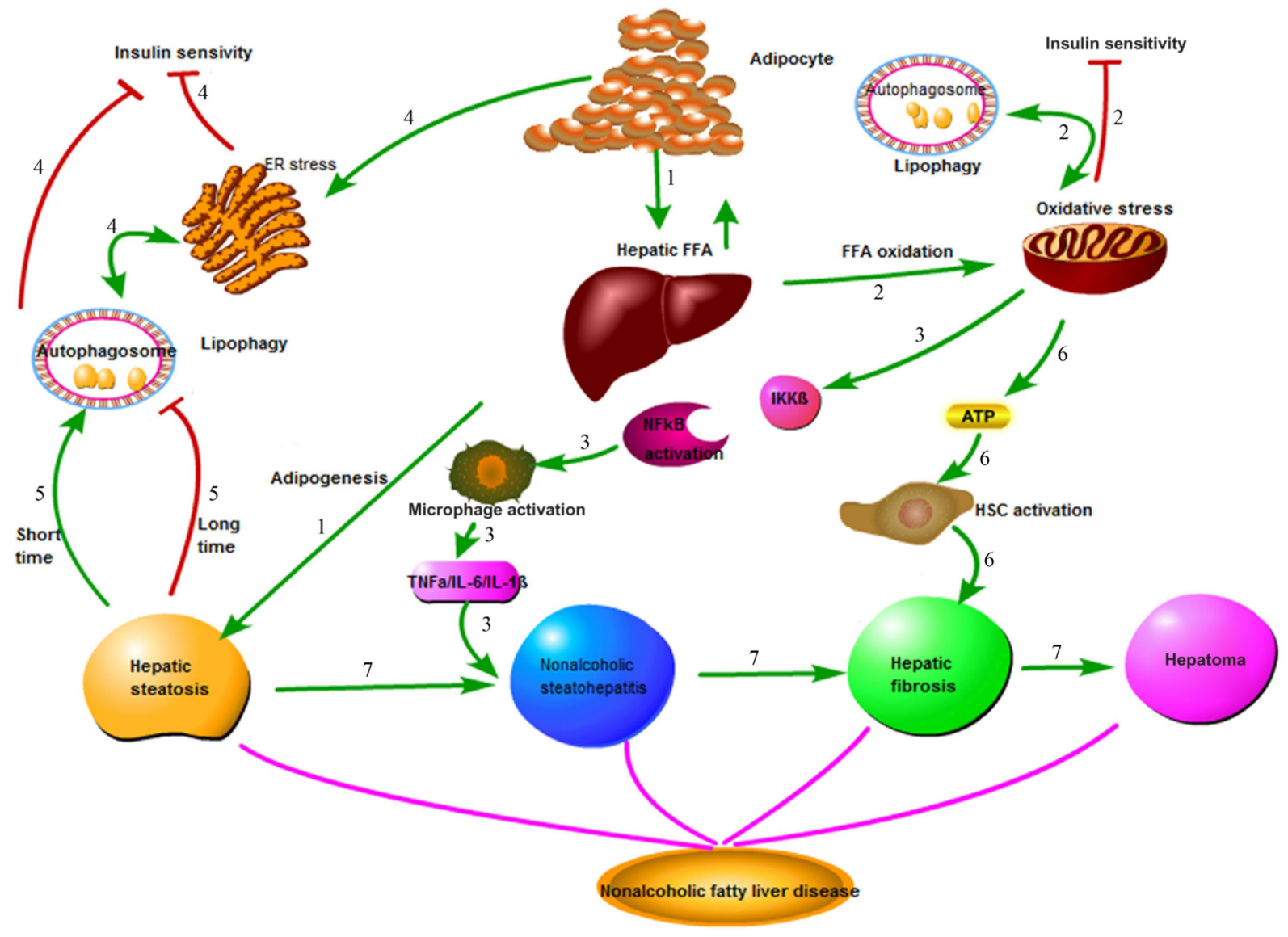

Figure I Pathophysiology of nonalcoholic fatty liver disease (NAFLD).

Notes: I. Adipocyte mobilization increases serum concentration of FFA and de novo synthesis of triglyceride. 2. Increased FFA oxidation in the mitochondria exceeds the capacity of the mitochondria, causing oxidative stress, which impairs insulin sensitivity and autophagy activation to digest defective proteins. 3 . Oxidative stress promotes phosphorylation and degradation of mesenchymal IKKB, accompanied by activated NF-KB translocation into the nucleus, causing inflammatory cascades and liver injury. 4. Obesity causes ER stress, which causes insulin resistance and stimulates autophagy due to an increase in unfolded proteins. Defective autophagy aggravates ER stress. 5. Short-term insulin resistance activates autophagy while long-term lipotoxicity impairs autophagy. 6. Energy produced by autophagy and oxidation fuels HSC activation, which promotes hepatic fibrosis. 7. NAFLD develops from steatosis, steatohepatitis, fibrosis, and hepatoma.

Abbreviations: FFA, free fatty acid; ER, endoplasmic reticulum; HSC, hepatic stellate cells.

\section{Autophagy}

Autophagy is a critical intracellular pathway that targets defective organelles to the lysosomes for degradation, and this process is highly preserved in eukaryotic cells. As an initial protective mechanism, autophagy is usually triggered by starvation or damage to maintain survival.

Three different types of autophagy are defined based on how the target substrates are delivered to the lysosomes for degradation: macroautophagy, microautophagy, and chaperone-mediated autophagy (CMA). ${ }^{7}$ Macroautophagy is the major type of autophagy. Initially, a small vascular sac called the isolation membrane is formed to enclose a portion of the cytoplasm or organelles, which results in the formation of a double-membrane structure named the autophagosome ${ }^{8}$ (Figure 2) The autophagosome fuses with the lysosome to form the autolysosome, which is involved in the degradation of defective or excessive organelles for energy recycling ${ }^{9}$ (Figure 3).
More than 30 autophagy-related genes (Atgs) have been discovered in yeast and play important roles in autophagy, many of which have mammalian homologs. ${ }^{10}$ Many molecules are involved in the formation and working process of autophagy. $\operatorname{Atg} 8 / \mathrm{LC} 3, \operatorname{Atg} 7$, and $\operatorname{Atg} 6 / \mathrm{Beclin}-1$ with the class III phosphoinositide 3-kinase (PI3K) complex are the best characterized. The process of autophagosome formation involves three major steps: 1) uncoordinated 51-like kinase (ULK) 1 helps in the double-membrane formation in the initiation phase; 2) nucleation with the Beclin-1/ class III PI3K complex; and 3) elongation of the isolation membrane with the help of LC3 lipidation. ${ }^{8,11}$ Conjugation of yeast Atg8 or mammalian LC3 with phosphatidylethanolamine (PE) during autophagy results in the formation of an insoluble form of $\operatorname{Atg} 8$ (Atg8-PE) or LC3 (LC3-II), which contributes to autophagosome formation. Atg8/LC3 stays on the membrane until it is degraded by lysosomes. 


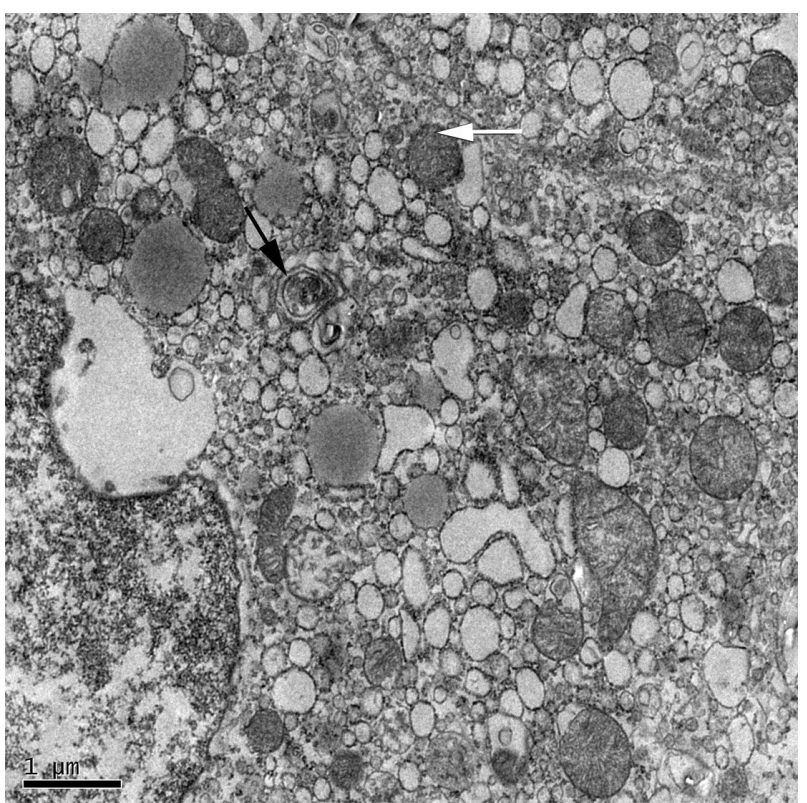

Figure 2 Electron micrograph showing ultrastructure of hepatocytes from a NAFLD mouse model.

Notes: Black arrow indicates autophagic vacuoles with a double-membrane structure that enclose lipid droplets and other defective organelles. White arrow shows autolysosome digesting contents. Original magnification: 100,000×.

Abbreviation: NAFLD, nonalcoholic fatty liver disease.

Therefore, $\operatorname{Atg} 8 / \mathrm{LC} 3$ is widely used as a marker for monitoring autophagy. ${ }^{12}$

Several signaling pathways are involved in the regulation of autophagy, among which, the class I PI3K/Akt/mTOR signaling pathway is the most classic one. PI3K promotes the phosphorylation of Akt, which subsequently leads to the activation of mTOR and the inhibition of autophagy. Conversely, inhibiting the activation of Akt induces a high level of autophagy, suggesting that class I PI3K/Akt/mTOR inversely regulates autophagy. ${ }^{13}$ When cells are under normal nutrient conditions, mTOR will be activated and phosphorylates ULK1, which subsequently combines with Atg13 and FIP200 and results in the inhibition of autophagosome formation (Figure 3). Rapamycin or nutrient deprivationinduced inhibition of mTOR leads to dephosphorylation of ULK1, ULK2, and Atg13, and activates ULK to phosphorylate FIP200, resulting in the stimulation of autophagy. ${ }^{14,15}$ Another drug named 3-methyladenine (3-MA) inhibits autophagy by suppressing the class III PI3K/Beclin-1 signaling pathway, which positively regulates autophagy. ${ }^{12}$ Furthermore, AMP-activated protein kinase (AMPK) plays a role in the regulation of autophagy. AMPK is activated by elevation of the AMP/ATP ratio ${ }^{12}$ in accordance with upregulation of autophagy under conditions of starvation, as described in Figure 3. Initial studies also found that AMPK is a positive regulator of autophagy both in the yeast ${ }^{16}$ and mammalian cells. ${ }^{17}$

\section{Autophagy and steatosis Autophagy reduces lipid droplets via lipophagy}

Several studies have demonstrated that autophagy is closely associated with metabolism, and this has caused considerable concern recently. Autophagy is characterized by digesting damaged organelles that are used to generate energy and materials for cell recycling. ${ }^{18}$ Degradation of cellular lipid stores through autophagy (lipophagy) is another way to degrade lipid besides cytosolic lipases, which opens up a new understanding of lipid degradation. ${ }^{19}$ Increased levels of fatty acids sustain the rates of mitochondrial $\beta$-oxidation for generation of ATP. ${ }^{20}$ However, excessive fatty acids will cause damage to mitochondria if they surpass the oxidation capacity of mitochondria. The increase in autophagy observed in a fatty liver can be confirmed by the presence of lipids enclosed within autophagic vacuoles and elevated expression of LC3-II, a bioprotein marker of autophagy.

Singh et al demonstrated that hepatocyte triglyceride content was significantly increased when challenged with fatty acid supplementation or cultured in methionine and choline-deficient medium with exogenous lipid supplementation treated with 3-MA, a pharmacological inhibitor of autophagy. ${ }^{19}$ Conversely, treatment with rapamycin, a pharmacological stimulator of autophagy, significantly decreases lipid droplets. ${ }^{19}$ It was reported recently that thyroid hormones, known to be important in promoting metabolism, probably stimulate fatty acid oxidation by induction of hepatic autophagy, which could be blocked by Atg 5 siRNA. ${ }^{21}$ Another study showed that treatment with caffeine reduced cellular lipid accumulation and the serum level of $\beta$-hydroxybutyrate, a product of lipolysis, and is accompanied with the increase of LC3-II. However, Atg5 knockdown in hepatocytes significantly increased intracellular lipids and reduced $\beta$-hydroxybutyrate levels. ${ }^{22}$ Another study demonstrated that pharmacological intervention with rapamycin or carbamazepine (mTORindependent inducer of autophagy) in high-fat diet (HFD)-fed mice obviously reduced hepatic and blood triglyceride, blood glucose and plasma insulin levels, ${ }^{23}$ indicating that modulation of autophagy alleviates NAFLD via an mTOR-dependent or -independent pathway. ${ }^{24}$ Autophagy removes lipid droplets not only in NAFLD but also in other factor-induced fatty liver diseases such as ethanol-induced steatosis. ${ }^{25}$

All the studies ${ }^{5,12,19,20}$ mentioned earlier showed similar results that intracellular reduction in lipid droplets was 


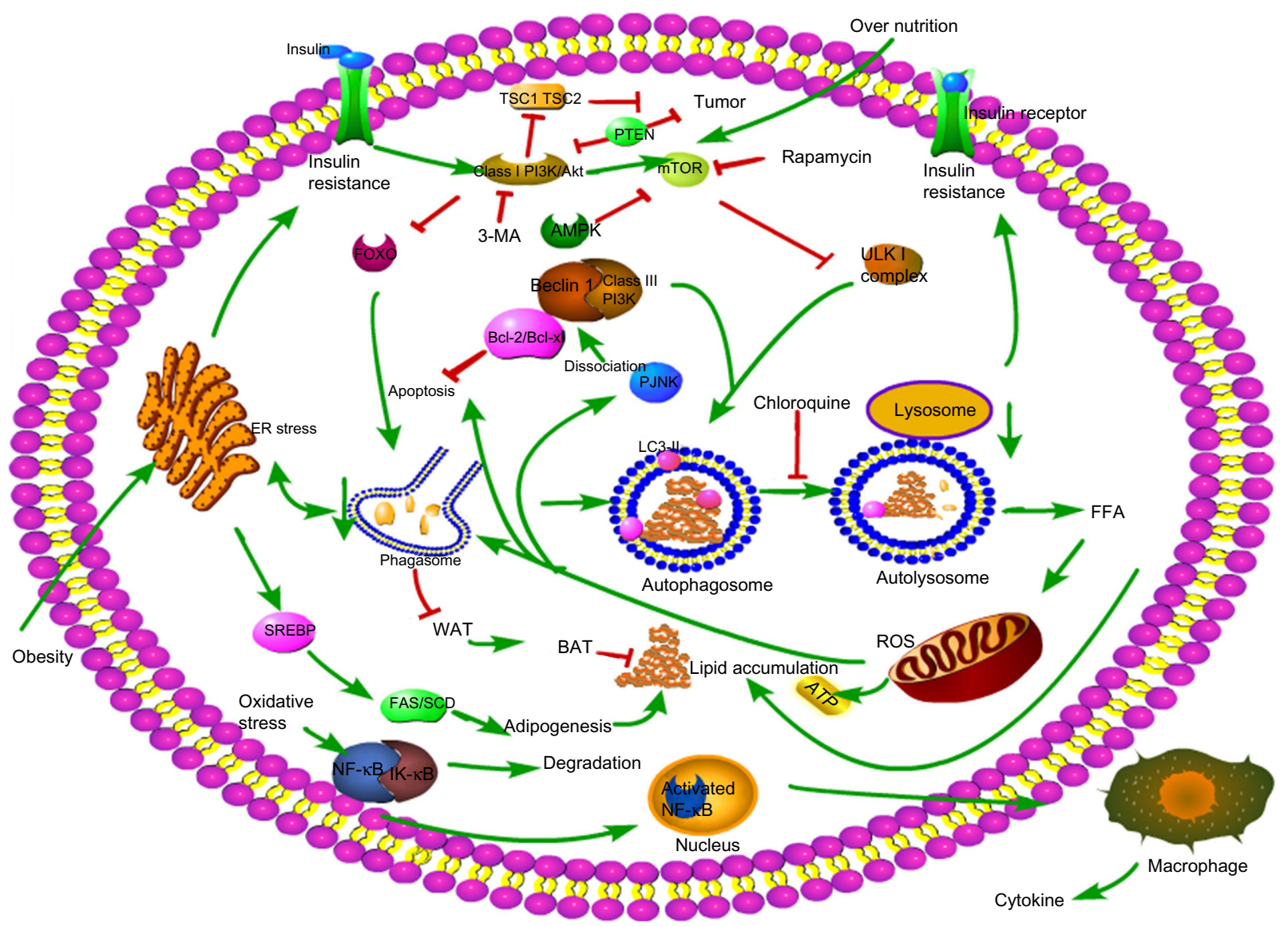

Figure 3 Molecular mechanisms involved in autophagy and NAFLD.

Notes: I. A small vascular sac called an isolation membrane is formed to enclose lipid droplets, which results in a double membrane structure called an autophagosome, which subsequently fuses with a lysosome to degrade lipid droplets into FFA. 2. Growth factor activates the PI3K/Akt signaling pathway to active mTOR, leading to phosphorylation of the ULKI complex, resulting in inhibition of autophagy formation. Short-term resistance may activate autophagy via inhibition of mTOR, while long-term lipotoxicity impairs autophagy by inhibition of the FOXO pathway. 3. The class III PI3K/Beclin-I complex positively regulates autophagy. 4. Increased FFA oxidation causes mitochondrial oxidative stress and activates autophagy. ROS also promotes cell apoptosis and JNK phosphorylation, which stimulates Bcl-2 phosphorylation and Bcl-2/ Beclin-I disassociation, resulting in apoptosis and autophagy, respectively. Likewise, ROS promotes NF- $\mathrm{KB}$ translocation into the nucleus, causing inflammatory cascades and liver injury. 5. Obesity causes ER stress, which activates autophagy and promotes adipogenesis-related gene expression such as FAS, SCD, leading to the synthesis of adipocytes. 6. Autophagy may show an inhibitory effect on adipocyte differentiation from WAT to BAT. 7. MP-activated protein kinase (AMPK) inhibits mTOR, and ultimately activates autophagy.

Abbreviations: NAFLD, nonalcoholic fatty liver disease; FFA, free fatty acid; ROS, reactive oxygen species; ER, endoplasmic reticulum; WAT, white adipose tissue; BAT, brown adipose tissue.

associated with a concomitant increase in autophagy flux, suggesting that autophagy plays essential roles in lipolysis, called lipophagy, which may provide a new way to eliminate fat in addition to lipase-mediated lipolysis. ${ }^{26,27}$ In fact, the effect of autophagy on lipid clearance may not be restricted to macrolipophagy, and indirect autophagy of organelles such as mitochondria and peroxisomes are probably involved; however, more investigations are needed to determine the independent effect of macrolipophagy. ${ }^{26}$

\section{Autophagy regulates adipogenesis and adipose tissue differentiation}

Contrary to the lipolysis effect, autophagy may also work on adipogenesis and lipocyte differentiation. It has been found that $\operatorname{Atg} 7$ knockdown of white adipose tissue (WAT) in mice showed a remarkable phenotype. The mutant mice were smaller than their wild-type counterparts, with larger numbers of smaller adipocytes filled with multilocular lipid droplets, as observed by light microscopy, and higher numbers of mitochondria, as observed by electron microscopy. All the results demonstrate that autophagy is critical for WAT adipogenesis, especially for the formation of the unilocular lipid droplet structure and for the mitochondria homeostasis control. ${ }^{28}$

Likewise, Ma et a ${ }^{29}$ discovered that deletion of FIP200, a core subunit of the mammalian autophagy related 1 complex, reduced both HFD- and starvation-induced steatosis and downregulated the gene expression of enzymes involved in de novo lipogenesis, which include ATP-citrate lyase (Acly), acetyl-CoA carboxylase (Acaca), fatty acid synthase (Fasn), and stearoyl-CoA desaturase-1 (Scd1). This was consistent 
with another study ${ }^{30}$ which reported that knockdown of $\operatorname{Atg} 7$ in pre-adipocytes inhibited lipid accumulation. Moreover, adipocyte-specific knockout of $\operatorname{Atg} 7$ generated lean mice with decreased WAT and increased brown adipocytes. ${ }^{30}$ Similar results were demonstrated by other studies where autophagy deficiency exerted a protective role against obesity by inhibiting adipogenesis. ${ }^{31-33}$ In addition to adipocytes, researchers using hepatocytes also demonstrated that intracellular lipid droplets were suppressed by $\operatorname{Atg} 7$ knockdown. ${ }^{34}$

Autophagy plays essential roles not only in adipogenesis but also in the process of WAT differentiation into mature tissue. Mouse embryonic fibroblasts with Atg5 deletion failed to complete the differentiation process, with the main cells developing multiple small lipid droplets and eventually dying. ${ }^{35}$ Although mitochondria are mostly responsible for fatty acid oxidation, their contribution to adipogenesis cannot be neglected. The number of mitochondria is increased in the early stage of adipogenesis and during maturation of WAT, due to the requirement of both energy and the key substrates for lipogenesis such as acetyl-CoA and glycerol 3-phosphate. However, the number of mitochondria was decreased by autophagy, called mitophagy, which may reflect the decreased need for lipogenesis in mature white adipocytes. The most significant features of adipose-specific Atg knockout in mice were an increased number of mitochondria and multiple small lipid droplets, compared with mature white adipocytes. ${ }^{36}$

Autophagy is also involved in the cell-type differentiation of adipocytes. WAT and brown adipose tissue (BAT) are two different types of tissue. In WAT, lipid storage prevents free fatty acid (FFA) lipotoxicity and provides energy when nutrients are restricted. BAT has a reduced capacity for lipid storage but higher rate of oxidation to provide energy. ${ }^{20}$ Tissue $\operatorname{Atg} 7$ knockout in mice had decreased WAT but increased BAT, along with an increased rate of oxidation and lean body mass, suggesting an inhibitory effect of autophagy on differentiation of WAT to BAT $^{30,37}$ (Figure 3).

Collectively, autophagy is involved in lipolysis, lipogenesis, and differentiation of adipocytes. The two mechanisms are not contradictory, but coexist and coact on lipid regulation. The imbalance between lipogenesis and lipolysis may result in steatosis, and studies focusing on expanding one side, such as lipolysis, may be of interest for NAFLD therapy.

\section{Molecular mechanisms linked with autophagy and steatosis}

Effect of lipotoxicity on autophagy varies with time

The effect of autophagy varies with time as well as nutritional status. Autophagy is activated by short-term stimulation of lipid availability as a self-protective mechanism to degrade lipid droplets. ${ }^{19}$ Both ob/ob mice and chronic HFD mice show markedly impaired hepatic autophagy, particularly expression of $\operatorname{Atg} 7 .{ }^{38}$ Furthermore, chronic exposure to lipid challenge has an inhibitory effect on CMA, which is probably associated with accelerated degradation of LAMP-2A. ${ }^{39}$ Liu et al found that long-term insulin resistance inhibited autophagy by inhibition of the constitutive nuclear form of FOXO1, which controls the transcription of autophagy-related genes, while short-term insulin resistance activates autophagy via the mTOR complex ${ }^{40}$ (Figure 3). Long-term lipid load may change membrane lipid composition and reduce autophagosome/lysosome fusion both in vitro and in vivo, which may partly explain why excessive lipid reduces autophagy and accelerates lipid accumulation. ${ }^{41}$

\section{Autophagy and aging}

Autophagy declines with aging in parallel with increased lipid accumulation in various organs, which may be associated with the increased prevalence of NAFLD, type II diabetes, and other metabolic disorders. The age-related changes in liver macroautophagic proteolysis have been extensively studied in vitro and in vivo. ${ }^{7}$ Both macroautophagy and CMA decrease with age. ${ }^{39,42}$ What is responsible for the decline in autophagy upon aging? Insulin/ IGF-1 is the best characterized pathway that is highly conserved in humans. ${ }^{43}$ Alterations in the IGF-1 signaling pathway may lead to extension of life and affect the process of autophagy. ${ }^{44}$ IGF-1 may inhibit macroautophagic proteolysis and degradation of mitochondria harboring deleterious mtDNA mutations. ${ }^{45}$ The IGF-1 signaling cascade includes PI3K/Akt pathway and is downstream of mTOR. Downregulation of PI3K/Akt stimulates autophagy, so autophagy may partly account for extension of life. ${ }^{13}$

\section{Autophagy and insulin resistance}

The pathogenesis of NAFLD involves a two-hit hypothesis. ${ }^{46}$ The first hit is insulin resistance, which is closely associated with the metabolic syndrome. Insulin resistance enhances lipolysis, increasing serum levels of FFA and decreasing very-low-density lipoprotein, which delivers triglycerides and cholesterol from the liver to peripheral organs, and promotes the resynthesis of lipid, thus leading to lipid storage in the liver. At the same time, fatty acid oxidation is increased and impairs mitochondrial oxidation, contributing to oxidative stress ${ }^{47}$ (Figure 1). As previously shown, insulin has an inhibitory effect on autophagy in the liver, but the mechanism remains unknown. This result is in agreement with a former 
study in which autophagy flux in rats treated with HFD for 2 weeks was upregulated, but was nearly inhibited after giving HFD for 10 weeks. ${ }^{48}$ FOXO transcription factors are regulators of longevity downstream of insulin signaling, which is involved in promoting expression of autophagy genes and the ubiquitin-proteasome system, known as two cell clearance processs. ${ }^{49}$ Another signaling pathway related to the inhibition of autophagy in the presence of insulin is class I PI3K/AKT that leads to the activation of mTOR and phosphorylation of its downstream p70S6k and autophagy inhibition, which can be blocked by rapamycin, an inhibitor of mTOR (Figure 3). ${ }^{50}$ Furthermore, Atg7 mutant mice showed impaired glucose tolerance, abnormal levels of insulin, and reduced $\beta$-cell mass. ${ }^{51}$ Defective hepatic autophagy promotes endoplasmic reticulum stress and causes insulin resistance. ${ }^{38}$

In contrast, autophagy deficiency in skeletal muscles leads to impaired activity of mitochondrial oxidative phosphorylation and subsequent induction of FGF-21 through ATF4 activation. As a result, autophagy deficiency enhanced oxidation and energy expenditure through browning of WAT and increased insulin sensitivity. ${ }^{32}$ In conclusion, the relationship between autophagy and insulin resistance is controversial. On the one hand, insulin resistance-induced steatosis reduces autophagy, which in turn aggravates insulin resistance and ER stress (Figure 3), and on the other hand, autophagy deficiency is involved in attenuating insulin resistance.

\section{Autophagy and steatohepatitis}

Benign hepatic steatosis can progress to nonalcoholic steatohepatitis (NASH), the state of inflammation and hepatocellular injury that is characterized by elevation of alanine aminotransferase, aspartate aminotransferase, and several adipokines and cytokines. Adiponectin, leptin, TNF- $\alpha$, and IL-6 play critical roles in the process of inflammation and liver injury ${ }^{52}$ (Figure 1).

Several studies have demonstrated that autophagy exhibits cellular protective effects, leading cells to survival from death stimuli. ${ }^{53-56}$ Cell damage is increased in long-term steatosis and aging in cells and tissues, suggesting that cell damage is correlated with autophagy reduction. Wang et al have discovered that autophagy inhibition by shRNA-mediated knockdown of Atg5 sensitizes cells to death from menadione by overactivation of $\mathrm{JNK}$ and the mitogen-activated protein kinase (MAPK)/extracellular signal-regulated kinase (ERK) $1 / 2$ signaling pathway. ${ }^{56}$

However, autophagy is a double-edged sword that mediates cell proliferation as well as cell death. Reactive oxygen species (ROS) produced by excessive FFA oxidation in mitochondria activate the JNK signaling pathway, promoting the dissociation of Bcl-2/Beclin-1 complex and autophagy induction, which is accompanied by apoptosis (Figure 3)..$^{57,58}$ The effect of autophagy on life and death of a cell is controversial.

\section{Immune activation and related signaling pathways}

Immune activation is a prerequisite for the development of $\mathrm{NASH}$, which exacerbates steatosis, including innate immune pathways. ${ }^{59}$ Salminen et al have discovered that inhibition of autophagic capacity with aging accelerates inflammation via activation of NLR P3. ${ }^{60}$

Activation of JNK and IKKB promotes the synthesis of AP-1 and NF- $\kappa B$, leading to an inflammatory reaction. ${ }^{59}$ Insulin resistance and oxidative stress stimulate phosphorylation and subsequent degradation of IKKB, accompanied by translocation of activated NF- $\kappa \mathrm{B}$ to the nucleus, causing inflammatory cascades, which in turn aggravate NF- $\mathrm{\kappa B}$ activation (Figure 1). Extracellular lipid can activate IKKB by engaging TLRs. Mice deficient in hepatocyte TLR4 attenuated severity of steatosis after challenge with an HFD, indicating that TLR4 is an important proinflammatory mediator. ${ }^{61}$ TLR may play an important role in pathogen-induced autophagy via a TRIF-dependent, MyD88-independent TLR4 signaling pathway, which has not been recognized before. ${ }^{62}$

Saitoh et al have shown that Atg 16L1 deficiency disrupts the formation of autophagosome, resulting in more severe damage and higher amounts of inflammatory cytokines such as IL-1 $\beta$ and IL-18, under stimulation with lipopolysaccharide. ${ }^{63}$ The mechanism by which autophagy regulates nonpathogen immunity, such as NASH, has not been elucidated and needs further investigation.

\section{Apoptosis}

Hepatocyte apoptosis due to FFA toxicity, called lipoapoptosis, is an important mechanism in the progression of NAFLD or NASH. ${ }^{64-66}$ Natarajan et al discovered that hepatocyte and cholangiocyte lipoapoptosis is induced by FFAs. ${ }^{66}$ Activation of p38-MAPK, ERK, and JNK signaling pathways is involved in the mechanism of lipoapoptosis, ${ }^{65,66}$ but the exact mechanism involved in the regulation of lipoapoptosis remains controversial.

Autophagy and apoptosis are two major ways of programmed cell death. There is significant cross talk between autophagy and apoptosis, as they can cooperate, antagonize, or assist each other under different conditions. ${ }^{67} \mathrm{MCL}-1$, an antiapoptotic Bcl-2 homolog, is a stress sensor that controls 
autophagy and apoptosis, and MCL-1 degradation not only induces apoptosis but also activates autophagy. ${ }^{68}$ It is established that the cross talk between apoptosis and autophagy is mediated, at least partially, by interaction of the Beclin-1 and $\mathrm{Bcl}-2$ complexes. ${ }^{69} \mathrm{Bcl}-2$ functions as an antiapoptotic protein as well as an anti-autophagy protein by inhibitory interaction with Beclin-1, to help maintain autophagy at levels that are compatible with cell survival rather than cell death. ${ }^{69}$ The connection between Bcl-2 and Beclin-1 or Bax is disrupted to induce autophagy and apoptosis, respectively, under conditions of stress. ${ }^{57}$ Localization of Bcl-2 to the ER prevents Beclin-1-mediated, starvation-regulated autophagy by binding to NAF-1, which stabilizes the interaction between Bcl-2 and Beclin-1 at the ER surface. ${ }^{70}$ Proapoptotic Bcl-2 homology (BH)3-only proteins have been described in previous studies to bind with the $\mathrm{BH} 3$-binding pocket of $\mathrm{Bcl}-2$ or $\mathrm{Bcl}-\mathrm{XL}$, leading to the disassociation of Bcl-2/XL and Beclin-1, resulting in autophagy or apoptosis, respectively. ${ }^{71,72}$ Activation of the JNK1 signaling pathway stimulates the disassociation of Bcl-2 from Beclin-1 via phosphorylating multiple residues at the $\mathrm{Bcl}-2$ regulatory position, leading to inactivated $\mathrm{Bcl}-2$ and autophagy induction ${ }^{65}$ (Figure 3 ).

There is another type of protein called death-associated protein kinase (DAPK) that is reported to promote disassociation of Bcl-2 from Beclin-1 by phosphorylating Thr119 of Beclin- $1{ }^{73} \mathrm{HMGB} 1$ is also suggested to be involved in the disassociation of Bcl-2 from Beclin-1. ${ }^{74,75}$ Autophagy is regarded as a cell survival mechanism; however, the interaction of Bcl-2 - an antiapoptotic protein -with Beclin-1 results in the inactivation of Beclin-1 and maintains autophagy to a certain extent. The disassociation of the complex leads to apoptosis and autophagy. The cross talk between autophagy and apoptosis is controversial and needs further investigation.

\section{Autophagy and liver fibrosis}

NAFLD is characterized by its course from simple steatosis to steatohepatitis, fibrosis, and cirrhosis. Hepatic fibrosis is the progressive accumulation of extracellular matrix that occurs in various chronic liver diseases, ${ }^{76}$ including NAFLD. Hepatic stellate cells (HSCs) function well in hepatic fibrosis, which may be a possible link with autophagy. HSC activation is critical in liver fibrosis, as it leads to the production of excessive extracellular matrix ${ }^{77}$ (Figure 1). According to a recent study, autophagy was increased in mice treated with $\mathrm{CCl}_{4}$ or thioacetamide, accompanied by lipid droplet loss and HSC activation. On the contrary, blockade of autophagy with siRNA, resulting in Atg5 knockdown, reduces fibrosis and matrix accumulation and increases the number and size of lipid droplets. ${ }^{78}$ Autophagy fuels tissue fibrogenesis not only in liver but also in other organs such as the lungs and kidneys, ${ }^{79}$ and may be a new factor in HSC activation. ${ }^{80}$ Autophagy affects the activation of HSCs not only due to the energy supply by autophagy, but also due to the effect of autophagy on cell growth cycle. 3-MA-induced autophagy inhibition is reported to block the G2 phase of HSC-T6 cells, a rat HSC line, leading to inhibition of cell proliferation. ${ }^{81}$ However, the relationship between autophagy and HSC proliferation remains controversial. An early study found that rapamycin an mTOR target inhibitor and autophagy stimulator-reduced fibrosis, ${ }^{82}$ and that this was due to its inhibition of cell proliferation or its indirect effect on autophagy induction; however, the mechanisms involved remain unclear.

Although autophagy plays essential roles in HSC activation and fibrosis promotion, its effect on antifibrosis and collagen degradation should not be neglected. In $\alpha$-1-antitrypsin deficiency, a disease in which the $\alpha$-1-antitrypsin mutant $Z$ protein results in protein aggregates and chronic liver injury, an autophagy-enhancing drug was demonstrated to reduce the hepatic load and reversed fibrosis. ${ }^{83}$ Both genetic and pharmacological inhibition of autophagy in mice resulted in increased levels of type I collagen in mouse kidneys and primary mesangial cells, suggesting that autophagy promotes intracellular degradation of type I collagen. ${ }^{84}$ However, whether it exhibits the same effect in liver remains unclear. Moreover, autophagy is regarded as a cell survival mechanism; it reduces oxidative stress and ER stress, and inhibits inflammation, thereby leading to fibrosis reduction.

We suggest that if autophagy could be selectively inhibited in HSCs and other fibrotic cells, a special blocker for autophagy would be an attractive candidate for antifibrotic strategies. However, this is challenging, as the tissue of the whole body is a complex of various cell types. Furthermore, since fibrosis is the result of an imbalance between collagen synthesis and degradation, the role of autophagy in collagen degradation is of great interest to the study of its antifibrotic mechanism.

\section{Autophagy and hepatic carcinogenesis}

NAFLD has the potential to progress into hepatic carcinoma. The role of autophagy in hepatic carcinogenesis is complex and is regarded as a double-edged sword ${ }^{85}$ Controversy exists as to whether autophagy inhibits or promotes carcinogenesis. Liang et al first reported that autophagy-related gene Beclin-1 was associated with cancer suppression. ${ }^{86}$ Several tumor suppressor genes such as PTEN, TSC1, and TSC2 stimulate 
autophagy by inhibiting mTOR pathway (Figure 3). On the contrary, class I PI3K/Akt activates oncogenes and inhibits autophagy by activating mTOR. ${ }^{11} \mathrm{Qu}$ et $\mathrm{al}{ }^{87}$ found that Beclin-1, a gene closely related to autophagy, was deleted in $40 \%-75 \%$ of the cases of human sporadic breast, ovarian, and prostate cancers. Beclin-1 gene knockout in mice increased the frequency of spontaneous hepatic malignancies and reduced autophagy. ${ }^{87}$ Similarly, it has been shown that autophagy may function in tumor suppression by mitigating metabolic stress and, in concert with apoptosis, by preventing death by necrosis. ${ }^{88}$ This evidence suggests that defective autophagy is positively correlated with carcinoma development.

However, autophagy is also a cell survival mechanism to help cells live through the environment of stress or nutrient restriction, thus leading to proliferation of tumor cells under stress, and may promote metastasis by enhancing tumor cell survival. ${ }^{89,90}$ Autophagy may suppress the p53 response by limiting DNA damage, oxidative stress, oncogene activation, and tumor cells less sensitive to starvation-induced cell death, thereby protecting cells from stress and promoting tumorigenesis. ${ }^{91}$ Furthermore, a recent study demonstrated that autophagy promoted HCC cell invasion by activation of epithelial-mesenchymal transition through a TGF- $\beta / \mathrm{Smad} 3$ signaling-dependent manner. ${ }^{92}$ In addition, LC3 is highly expressed in hepatoma compared with normal tissues, which is regarded as an independent predictor of hepatoma recurrence after surgery. ${ }^{93}$

As yet, it is undetermined as to whether autophagy promotes or suppresses carcinoma. The double-edged sword effect of autophagy on tumors makes it important as a potential therapeutic option for cancer suppression, if properly controlled. However, several important questions are of concern. How long does it take for autophagy inhibition or activation to suppress an established tumor? Does the inhibition or activation effect depend on the tumor type or the drug concentration? How does autophagy function in tumors deficient in autophagy-related genes? In summary, activation of autophagy may represent a promising and effective strategy for liver cancer therapy, while the inhibition of autophagy may lead to the death of malignant cells due to energy restriction. ${ }^{94}$ Further investigations are needed to determine the dual role of autophagy in carcinoma.

\section{Future perspectives and conclusion}

Autophagy is associated with NAFLD. Much work is needed to determine the exact relationship between autophagy and NAFLD, and the results from various studies are controversial. Most studies have provided evidence that autophagy deficiency leads to steatosis, since autophagy digests lipid droplets, and drugs that increase autophagy may be a potential therapeutic approach in NAFLD.

However, several studies have demonstrated that autophagy is essential in adipogenesis and differentiation. Whether the difference in the results is related to the extent of autophagy or activation of other signaling pathways needs further investigation. It may also be significant to focus on other cell types such as HSCs and Kupffer cells, which are also involved in the progression of NAFLD ${ }^{95}$ in addition to hepatocytes. More attention should be paid to the contribution of other types of autophagy to the pathogenesis of NAFLD; for example, CMA, though macroautophagy is regarded as the major type of autophagy.

There are some other questions that deserve consideration. Is the mechanism of lipid regulation tissue specific in the liver? Upregulation of autophagy protects hepatocytes against lipotoxicity, but cancer may also be promoted since autophagy is regarded as a cellular survival mechanism and helps cells to live through the conditions of stress and nutrient restriction. The answer to whether autophagy is a friend or foe in cancer is controversial. Moreover, the cross talk between apoptosis and autophagy, and the extent autophagy provides a protective effect in cells, need to be investigated. Autophagy induces HSC activation and stimulates hepatic fibrosis; however, autophagy fuels HSC activation and works on collagen degradation and inflammation inhibition, which seems to inhibit fibrosis. It is unclear as to whether the different effects of autophagy on fibrosis are cell type- or tissue-specific. The selective inhibitor of autophagy may be an ideal candidate for antifibrotic medicines.

In conclusion, a better understanding of the bidirectional characters of autophagy and its regulatory mechanisms may allow the practical use of autophagy for therapeutic purposes in the future.

\section{Acknowledgment}

Supported by Research Grant for Key Clinical Discipline Construction of Shanghai Municipality, People's Republic of China (grant number: ZK2012B20).

\section{Disclosure}

The authors report no conflicts of interest in this work.

\section{References}

1. Chalasani N, Younossi Z, Lavine JE, et al. The diagnosis and management of non-alcoholic fatty liver disease: practice guideline by the American Association for the Study of Liver Diseases, American College of Gastroenterology, and the American Gastroenterological Association. Hepatology. 2012;55:2005-2023. 
2. Milic S, Stimac D. Nonalcoholic fatty liver disease/steatohepatitis: epidemiology, pathogenesis, clinical presentation and treatment. Dig Dis. 2012; 30:158-162.

3. Birkenfeld AL, Shulman GI. Non alcoholic fatty liver disease, hepatic insulin resistance and type 2 diabetes. Hepatology. 2013:59:713-723.

4. Kim D, Choi SY, Park EH et al. Nonalcoholic fatty liver disease is associated with coronary artery calcification. Hepatology. 2012;56:605-613.

5. Sinha RA, Farah BL, Singh BK, et al. Caffeine stimulates hepatic lipid metabolism by the autophagy-lysosomal pathway in mice. Hepatology 2014;59:1366-1380.

6. Amir M, Czaja MJ. Autophagy in nonalcoholic steatohepatitis. Expert Rev Gastroenterol Hepatol. 2011;5:159-166.

7. Cuervo AM, Bergamini E, Brunk UT, Droge W, French M, Terman A. Autophagy and aging: the importance of maintaining "clean" cells Autophagy. 2005;1:131-140.

8. Rautou PE, Mansouri A, Lebrec D, Durand F, Valla D, Moreau R. Autophagy in liver diseases. J Hepatol. 2010;53:1123-1134.

9. Mizushima N, Yoshimori T, Ohsumi Y. The role of Atg proteins in autophagosome formation. Annu Rev Cell Dev Biol. 2011;27: 107-132.

10. Mizushima N, Yoshimori T, Levine B. Methods in mammalian autophagy research. Cell. 2010;140:313-326.

11. Levine B, Kroemer G. Autophagy in the pathogenesis of disease. Cell. 2008;132:27-42.

12. Yin XM, Ding WX, Gao W. Autophagy in the liver. Hepatology 2008;47:1773-1785

13. Shintani T, Klionsky DJ. Autophagy in health and disease: a doubleedged sword. Science. 2004;306:990-995.

14. Murrow L, Debnath J. Autophagy as a stress-response and qualitycontrol mechanism: implications for cell injury and human disease. Annu Rev Pathol. 2013;8:105-137.

15. Jung CH, Jun CB, Ro SH, et al. ULK-Atg13-FIP200 complexes mediate mTOR signaling to the autophagy machinery. Mol Biol Cell. 2009;20:1992-2003

16. Wang Z, Wilson WA, Fujino MA, Roach PJ. Antagonistic controls of autophagy and glycogen accumulation by Snflp, the yeast homolog of AMP-activated protein kinase, and the cyclin-dependent kinase Pho85p. Mol Cell Biol. 2001;21:5742-5752.

17. Takagi H, Matsui Y, Hirotani S, Sakoda H, Asano T, Sadoshima J. AMPK mediates autophagy during myocardial ischemia in vivo. Autophagy. 2007;3:405-407.

18. Rabinowitz JD, White E. Autophagy and metabolism. Science. 2010: 330:1344-1348.

19. Singh R, Kaushik S, Wang Y, et al. Autophagy regulates lipid metabolism. Nature. 2009;458:1131-1135.

20. Liu K, Czaja MJ. Regulation of lipid stores and metabolism by lipophagy. Cell Death Differ. 2013;20:3-11.

21. Sinha RA, You SH, Zhou J, et al. Thyroid hormone stimulates hepatic lipid catabolism via activation of autophagy. J Clin Invest 2012;122:2428-2438

22. Sinha RA, Farah BL, Singh BK, et al. Caffeine stimulates hepatic lipid metabolism by the autophagy-lysosomal pathway in mice. Hepatology. 2014;59:1366-1380

23. Lin CW, Zhang H, Li M, et al. Pharmacological promotion of autophagy alleviates steatosis and injury in alcoholic and non-alcoholic fatty liver conditions in mice. J Hepatol. 2013;58:993-999.

24. Gracia-Sancho J, Guixe-Muntet, S, Hide D, Bosch J. Modulation of autophagy for the treatment of liver diseases. Expert Opin Investig Drugs. 2014;23:965-977.

25. Ding WX, LiM, Chen X, et al. Autophagy reduces acute ethanol-induced hepatotoxicity and steatosis in mice. Gastroenterology. 2010;139: 1740-1752.

26. Zechner R, Madeo F. Cell biology: another way to get rid of fat. Nature. 2009;458:1118-1119.

27. Mao Y, Cheng J, Yu F, Li H, Guo C, Fan X. Ghrelin attenuated lipotoxicity via autophagy induction and nuclear factor-kappaB inhibition. Cell Physiol Biochem. 2015;37:563-576.
28. Goldman S, Zhang Y, Jin S. Autophagy and adipogenesis: implications in obesity and type II diabetes. Autophagy. 2010;6:179-181.

29. Ma D, Molusky MM, Song J, et al. Autophagy deficiency by hepatic FIP200 deletion uncouples steatosis from liver injury in NAFLD. $\mathrm{Mol}$ Endocrinol. 2013;27:1643-1654.

30. Singh R, Xiang Y, Wang Y, et al. Autophagy regulates adipose mass and differentiation in mice. J Clin Invest. 2009;119:3329-3339.

31. Zhang Y, Goldman S, Baerga R, Zhao Y, Komatsu M, Jin S. Adiposespecific deletion of autophagy-related gene 7 (atg7) in mice reveals a role in adipogenesis. Proc Natl Acad Sci US A. 2009;106:19860-19865.

32. Kim KH, Jeong YT, Oh H, et al. Autophagy deficiency leads to protection from obesity and insulin resistance by inducing Fgf 21 as a mitokine. Nat Med. 2013;19:83-92.

33. Lapierre LR, Silvestrini MJ, Nunez L, et al. Autophagy genes are required for normal lipid levels in C. elegans. Autophagy. 2013;9:278-286.

34. Shibata M, Yoshimura K, Furuya N, et al. The MAP1-LC3 conjugation system is involved in lipid droplet formation. Biochem Biophys Res Commun. 2009;382:419-423.

35. Baerga R, Zhang Y, Chen PH, Goldman S, Jin S. Targeted deletion of autophagy-related 5 (atg5) impairs adipogenesis in a cellular model and in mice. Autophagy. 2009;5:1118-1130.

36. Goldman SJ, Zhang Y, Jin S. Autophagic degradation of mitochondria in white adipose tissue differentiation. Antioxid Redox Signal. 2011;14:1971-1978.

37. Singh R. Autophagy and regulation of lipid metabolism. Results Probl Cell Differ. 2010;52:35-46.

38. Yang L, Li P, Fu S, Calay ES, Hotamisligil GS. Defective hepatic autophagy in obesity promotes ER stress and causes insulin resistance. Cell Metab. 2010;11:467-478.

39. Rodriguez-Navarro JA, Kaushik S, Koga H, et al. Inhibitory effect of dietary lipids on chaperone-mediated autophagy. Proc Natl Acad Sci U S A. 2012;109:E705-E714.

40. Liu HY, Han J, Cao SY, et al. Hepatic autophagy is suppressed in the presence of insulin resistance and hyperinsulinemia: inhibition of FoxO1-dependent expression of key autophagy genes by insulin. J Biol Chem. 2009:284:31484-31492.

41. Koga H, Kaushik S, Cuervo AM. Altered lipid content inhibits autophagic vesicular fusion. Faseb J. 2010;24:3052-3065.

42. Cuervo AM, Dice JF. Age-related decline in chaperone-mediated autophagy. J Biol Chem. 2000;275:31505-31513.

43. Longo VD, Finch CE. Evolutionary medicine: from dwarf model systems to healthy centenarians? Science. 2003;299:1342-1346.

44. Brown-Borg HM, Borg KE, Meliska CJ, Bartke A. Dwarf mice and the ageing process. Nature. 1996;384:33.

45. Gu Y, Wang C, Cohen A. Effect of IGF-1 on the balance between autophagy of dysfunctional mitochondria and apoptosis. Febs Lett. 2004;577:357-360.

46. Day CP, James OF. Steatohepatitis: a tale of two "hits"? Gastroenterology. 1998;114:842-845.

47. Kawano Y, Cohen DE. Mechanisms of hepatic triglyceride accumulation in non-alcoholic fatty liver disease. J Gastroenterol. 2013;48: 434-441.

48. Papackova Z, Dankova H, Palenickova E, Kazdova L, Cahova M. Effect of short- and long-term high-fat feeding on autophagy flux and lysosomal activity in rat liver. Physiol Res. 2012;61(Suppl 2): S67-S76.

49. Webb AE, Brunet A. FOXO transcription factors: key regulators of cellular quality control. Trends Biochem Sci. 2014;39:159-169.

50. Kanazawa T, Taneike I, Akaishi R, et al. Amino acids and insulin control autophagic proteolysis through different signaling pathways in relation to mTOR in isolated rat hepatocytes. $J$ Biol Chem. 2004;279:8452-8459.

51. Ebato C, Uchida T, Arakawa M, et al. Autophagy is important in islet homeostasis and compensatory increase of beta cell mass in response to high-fat diet. Cell Metab. 2008;8:325-332.

52. Pinzani M. Pathophysiology of non-alcoholic steatohepatitis and basis for treatment. Dig Dis. 2011;29:243-248. 
53. Pua HH, Dzhagalov I, Chuck M, Mizushima N, He YW. A critical role for the autophagy gene Atg5 in T cell survival and proliferation. J Exp Med. 2007;204:25-31.

54. Fukuo Y, Yamashina S, Sonoue H, et al. Abnormality of autophagic function and cathepsin expression in the liver from patients with nonalcoholic fatty liver disease. Hepatol Res. 2014;44(9):1026-1036.

55. Wu D, Cederbaum AI. Inhibition of autophagy promotes CYP2E1dependent toxicity in HepG2 cells via elevated oxidative stress, mitochondria dysfunction and activation of $\mathrm{p} 38$ and JNK MAPK. Redox Biol. 2013;1:552-565.

56. Wang Y, Singh R, Xiang Y, Czaja MJ. Macroautophagy and chaperonemediated autophagy are required for hepatocyte resistance to oxidant stress. Hepatology. 2010;52:266-277.

57. Mukhopadhyay S, Panda PK, Sinha N, Das DN, Bhutia SK. Autophagy and apoptosis: where do they meet? Apoptosis. 2014;19:555-566.

58. Youle RJ, Narendra DP. Mechanisms of mitophagy. Nat Rev Mol Cell Biol. 2011;12:9-14.

59. Maher JJ, Leon P, Ryan JC. Beyond insulin resistance: Innate immunity in nonalcoholic steatohepatitis. Hepatology. 2008;48:670-678.

60. Salminen A, Kaarniranta K, Kauppinen A. Inflammaging: disturbed interplay between autophagy and inflammasomes. Aging (Albany NY). 2012;4:166-175.

61. Jia L, Vianna CR, Fukuda M, et al. Hepatocyte Toll-like receptor 4 regulates obesity-induced inflammation and insulin resistance. Nat Commun.2014;5:3878.

62. XuY, Jagannath C, Liu XD, Sharafkhaneh A, Kolodziejska KE, Eissa NT. Toll-like receptor 4 is a sensor for autophagy associated with innate immunity. Immunity. 2007;27:135-144.

63. Saitoh T, Fujita N, Jang MH, et al. Loss of the autophagy protein Atg16L1 enhances endotoxin-induced IL-1beta production. Nature. 2008;456:264-268.

64. Mei S, Ni HM, ManleyS, et al. Differential roles of unsaturated and saturated fatty acids on autophagy and apoptosis in hepatocytes. J Pharmacol Exp Ther. 2011;339:487-498.

65. Komiya K, Uchida T, Ueno T, et al. Free fatty acids stimulate autophagy in pancreatic beta-cells via JNK pathway. Biochem Biophys Res Commun. 2010;401:561-567.

66. Natarajan SK, Ingham SA, Mohr AM, et al. Saturated free fatty acids induce cholangiocyte lipoapoptosis. Hepatology. 2014;60(6): 1942-1956.

67. Nikoletopoulou V, Markaki M, Palikaras, K, Tavernarakis N. Crosstalk between apoptosis, necrosis and autophagy. Biochim Biophys Acta. 2013; 1833:3448-3459.

68. Germain M, Nguyen AP, Le Grand JN, et al. MCL-1 is a stress sensor that regulates autophagy in a developmentally regulated manner. Embo J. 2011;30:395-407.

69. Pattingre S, Tassa A, Qu X, et al. Bcl-2 antiapoptotic proteins inhibit Beclin 1-dependent autophagy. Cell.2005;122:927-939.

70. Chang NC, Nguyen M, Germain M, Shore GC. Antagonism of Beclin 1-dependent autophagy by BCL-2 at the endoplasmic reticulum requires NAF-1. Embo J. 2010;29:606-618.

71. Oberstein A, Jeffrey PD, Shi Y. Crystal structure of the Bcl-XL-Beclin 1 peptide complex: Beclin 1 is a novel BH3-only protein. $J$ Biol Chem. 2007;282:13123-13132.

72. Feng W, Huang S, Wu H, Zhang M. Molecular basis of Bcl-xL's target recognition versatility revealed by the structure of $\mathrm{Bcl}-\mathrm{xL}$ in complex with the BH3 domain of Beclin-1. J Mol Biol. 2007;372:223-235.

73. Zalckvar E, Berissi H, Mizrachy L, Iet al. DAP-kinase-mediated phosphorylation on the $\mathrm{BH} 3$ domain of beclin 1 promotes dissociation of beclin 1 from Bcl-XL and induction of autophagy. Embo Rep. 2009; 10:285-292.
74. Kang R, Livesey KM, Zeh HJ, Loze MT, Tang D. HMGB1: a novel Beclin 1-binding protein active in autophagy. Autophagy. 2010;6:1209-1211.

75. Shen M, Lu J, Dai W, et al. Ethyl pyruvate ameliorates hepatic ischemiareperfusion injury by inhibiting intrinsic pathway of apoptosis and autophagy. Mediators Inflamm. 2013;2013:461-536.

76. Moreno M, Chaves JF, Sancho-Bru P, et al. Ghrelin attenuates hepatocellular injury and liver fibrogenesis in rodents and influences fibrosis progression in humans. Hepatology. 2010;51:974-985.

77. Friedman SL. Mechanisms of hepatic fibrogenesis. Gastroenterology. 2008:134:1655-1669.

78. Hernandez-Gea V, Ghiassi-Nejad Z, Rozenfeld R, et al. Autophagy releases lipid that promotes fibrogenesis by activated hepatic stellate cells in mice and in human tissues. Gastroenterology. 2012;142:938-946.

79. Hernandez-Gea V, Friedman SL. Autophagy fuels tissue fibrogenesis. Autophagy. 2102;8:849-850.

80. Thoen LF, Guimaraes EL, Grunsven LA. Autophagy: a new player in hepatic stellate cell activation. Autophagy. 2102;8:126-128.

81. Liu M, He Y, Zhang J. [Effect of autophagy inhibitor 3-methyladenine on proliferation and activation of hepatic stellate cells]. Xi Bao Yu Fen Zi Mian Yi Xue Za Zhi. 2103;29:809-812. Chinese.

82. Zhu J, Wu J, Frizell E, et al. Rapamycin inhibits hepatic stellate cell proliferation in vitro and limits fibrogenesis in an in vivo model of liver fibrosis. Gastroenterology. 1999;117:1198-1204.

83. Hidvegi T, Ewing M, Hale P, et al. An autophagy-enhancing drug promotes degradation of mutant alpha1-antitrypsin $\mathrm{Z}$ and reduces hepatic fibrosis. Science. 2010;329:229-232.

84. Kim SI, Na HJ, Ding Y, Wang Z, Lee SJ, Choi ME. Autophagy promotes intracellular degradation of type I collagen induced by transforming growth factor (TGF)-beta1. J Biol Chem. 2012;287:11677-11688.

85. White E, DiPaola RS. The double-edged sword of autophagy modulation in cancer. Clin Cancer Res. 2009;15:5308-5316.

86. Liang XH, Jackson S, Seaman M, et al. Induction of autophagy and inhibition of tumorigenesis by beclin 1. Nature. 1999;402:672-676.

87. Qu X, Yu J, Bhagat G, et al. Promotion of tumorigenesis by heterozygous disruption of the beclin 1 autophagy gene. J Clin Invest. 2003;112:1809-1820.

88. Degenhardt K, Mathew R, Beaudoin B, et al. Autophagy promotes tumor cell survival and restricts necrosis, inflammation, and tumorigenesis. Cancer Cell. 2006;10:51-64.

89. Bhutia SK, Mukhopadhyay S, Sinha N, et al. Autophagy: cancer's friend or foe? Adv Cancer Res. 2013;118:61-95.

90. Yang $\mathrm{S}$, Wang $\mathrm{X}$, Contino $\mathrm{G}$, et al. Pancreatic cancers require autophagy for tumor growth. Genes De. 2011;25:717-729.

91. Guo JY, Xia B, White E. Autophagy-mediated tumor promotion. Cell. 2013;155:1216-1219.

92. Li J, Yang B, Zhou Q, et al. Autophagy promotes hepatocellular carcinoma cell invasion through activation of epithelial-mesenchymal transition. Carcinogenesis. 2013;34:1343-1351.

93. Yang JD, Seol SY, Leem SH, et al. Genes associated with recurrence of hepatocellular carcinoma: integrated analysis by gene expression and methylation profiling. J Korean Med Sci. 2011;26:1428-1438.

94. Lavallard VJ, Gual P. Autophagy and non-alcoholic fatty liver disease. Biomed Res Int. 2014;2014:120-179.

95. Baratta JL, Ngo A, Lopez B, Kasabwalla N, Longmuir KJ, Robertson RT. Cellular organization of normal mouse liver: a histological, quantitative immunocytochemical, and fine structural analysis. Histochem Cell Biol. 2009;131:713-726. 
Hepatic Medicine: Evidence and Research

Dovepress

\section{Publish your work in this journal}

Hepatic Medicine: Evidence and Research is an international, peerreviewed, open access journal covering all aspects of adult and pediatric hepatology in the clinic and laboratory including the following topics: Pathology, pathophysiology of hepatic disease; Investigation and treatment of hepatic disease; Pharmacology of drugs used for the treatment of hepatic disease. Issues of patient safety and quality of care will also be considered. The manuscript management system is completely online and includes a very quick and fair peer-review system, which is all easy to use. Visit http://www.dovepress.com/ testimonials.php to read real quotes from published authors.

Submit your manuscript here: http://www.dovepress.com/hepatic-medicine-evidence-and-research-journal 